\title{
432-MHz RF Source For The JHP Proton Linac
}

\author{
M. ONO, S. ANAMI, H. HANAKI, Z. IGARASHI, M. KAWAMURA, T. KUBO, \\ C. KUBOTA, K. KUDO, E. TAKASAKI and T. TAKENAKA \\ National Laboratory for High Energy Physics(KEK) \\ 1-1 Oho,Tsukuba-shi,Ibaraki-ken,305,Japan
}

\section{Abstract}

A high duty UHF(432-MHz) RF source for the intense proton linac of the Japanese Hadron Project (JHP) has been constructed at KEK. A test-linac consisting of a RFQ and a drift-tube (DTL) structures is under construction. The UHF $\mathrm{RF}$ power generated by $2-\mathrm{MW}$ peak power klystron will be fed into these structures with $650 \mu \mathrm{sec}$ pulse-duration and 50 $\mathrm{Hz}$. repetition-rate through an waveguide system with a $\mathrm{Y}$-junction circulator. A dc high voltage (max.-95 kV) is applied to a cathode of the klystron and a beam current is modulated up to $40 \mathrm{~A}$ by a pulsed high voltage (max. $80 \mathrm{kV})$ applied to a modulating anode. The klystron power supply can operate two such klystrons simultaneously with two individual anode modulators. The first operation of this RF power source and the preliminary test of an RF signal processing such as PLL and ALC will be reported.

\section{INTRODUCTION}

The proton linac of the JHP[1] has been designed to have three types of accelerating structures such as an RFQ, a DTL and a coupled-cell structure. The test-linac consisting of the former two linacs and a proton-source $\left(\mathrm{H}^{-}\right)$is under construction to prove a feasibility of the intense proton beam linac required for the JHP. The UHF(432-MHz) RF sources for the $R \Gamma Q$ and the DTL will be generated by the two high-power, high-duty klystrons with $650 \mu \mathrm{sec}$ pulse-duration and $50 \mathrm{~Hz}$ repetition-rate. The klystron power supply can feed electricpower to two klystrons with a same cathode voltage but with two individual modulating anode controls. The first operation of the klystron generating 2-MW peak power with $600 \mu \mathrm{sec}$ pulse-duration and the basic test of the ALC (automatic level control) within the pulse duration has been done. The high power feed to the cavities with the needed RF processing such as ALC and PLL (phase lock loop) will soon start.

\section{RF SYSTEM}

\section{A. Klystron}

The RF power required from the test-linac is $\sim 1 \mathrm{MW} /$ klystron with $\sim 3 \%$ duty $(650 \mu \mathrm{sec} \times 50 \mathrm{~Hz})$. Within the pulse duration the RF processing such as ALC and PLL are expected. Not only such feedback processing, some kind of feedforward processing is also expected to compensate the heavy beam loading for every pulse duration. The klystron can't be operated at a saturation to cope with such requirements. The installed klystron (TH2134, THOMSON-CSF) can produce a 2-MW peak power at the saturation with the required duty, and it will be operated in a linear region with a reduced power to realize the needed RF processing. Such a capability of generating an excessive power may also ensure a stable operation of these high-power and high-duty system. The nominal cathodeand anode-voltages are $-93 \mathrm{kV}$ and $80 \mathrm{kV}$ respectively to generate $2-\mathrm{MW}$ power with a $40 \mathrm{~A}$ beam current.

\section{B. Power Supply}

The scheme of the klystron power supply is shown in Figure 1. The power supply is able to operate two klystrons simultancously. It generate maximum $-110 \mathrm{kV}$ cathode voltage with $46 \mathrm{~A} \times 2$ beam current at $650 \mu \mathrm{sec}$ pulse-duration and $50 \mathrm{~Hz}$ repetition rate. Thyristor switches control and stabilize the cathode voltage. With allowing $5 \%$ sag at the above condition, $10.8 \mu \mathrm{F}$ filter condenser is chosen. A crowbar switch consisting of 6-series ignitrons (Richardson Elect., NL35391 ) is installed for klystron protection. If the crowbar is triggered the thyristor switch will reduce a flow-in electric power within $\sim 5 \mathrm{msec}$ and then $\mathrm{VCB}$ is switched off at $\sim 20 \mathrm{msec}$. A series-resister is installed in the tank just before of the klystron cathode to suppress a flash-over current. The crowbar is also set aside the tank to minimize a cable length. In order to check the crowbar switch, the cathode-voltageshortening-tests were carried out using a thin copper wire (Ø0.35) shortened at the klystron socket. Due to the adopted $27.5 \Omega$ series-resister and the fast crowbar work (less than 6 $\mu \mathrm{sec}$ ) an estimated deposit energy at the wire is much less than 10 Joule at $-91 \mathrm{kV}$ cathode voltage. The estimation is carried out by using a measured shortening current and an estimated wire resistance.
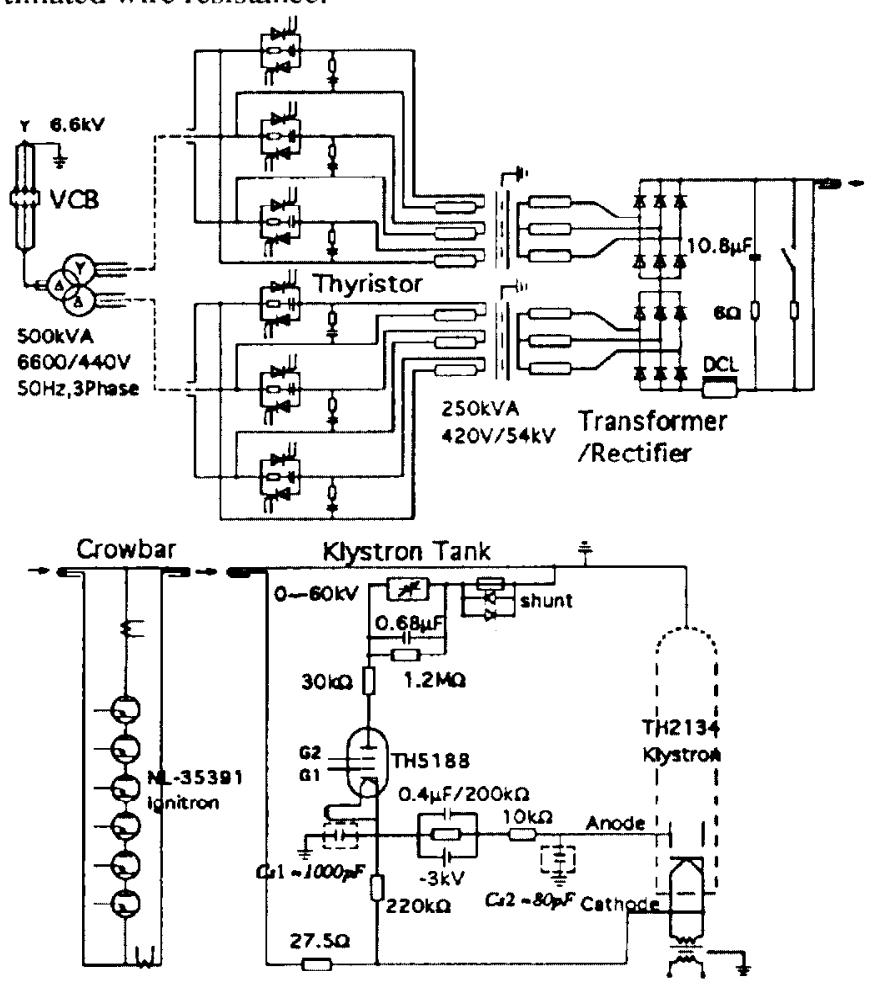

Figure 1. Klystron Power Supply.

\section{Anode Modulation}

The klystron beam current is modulated by the anode voltage produced by the switching tube (TH5188, THOMSON 
-CSF) with the resister-chain divider. At the switch-on of this tube, the observed current which flow across the tube, was several hundreds $\mathrm{mA}$ consisting of $\sim 160 \mathrm{~mA}$ anode-current of the klystron and $\sim 360 \mathrm{~mA}$ current through the $220 \mathrm{k} \Omega$, in the 2-MW output operation. The grid-pulse of the switching tube is easily controlled by a low level signal through light guide cable. The control units of $\mathrm{G} 1$ and $\mathrm{G} 2$ of the switching tube arc all set in a box, and this box is connected to the cathode voltage's potential. The stray capacitor that is estimated from the box configuration in the tank is relatively large. Even though this large capacitor is responsible to a large rise- and fall-time of the anode voltage, we adopted this scheme because of its rather simple and reliable method. A clamp voltage ( 0 to $-60 \mathrm{kV}$ ) is prepared between the ground and the resister-chain to control anode voltage in the range defined as follow,

$$
V_{\text {Anode }}=-\left(V_{\text {Cathode }}-V_{\text {Clamp }}\right) \times R_{2} /\left(R_{1}+R_{2}\right)+V_{\text {Bias }} \text {, }
$$

where $R_{1}$ and $R_{2}$ are resistors shown as $30 \mathrm{k} \Omega$ and $220 \mathrm{k} \Omega$ in Figure 1 , and $V_{B i a s}(-3 \mathrm{kV})$ is a bias voltage to secure the cutoff statc of the klystron at the inter-pulse. Comments are in order. If the average current which flow across the resisterchain exceeds the clamp-power-supply-current expressed as $V_{\text {Clamp }} / 1.2 \mathrm{M} \Omega$, the clamp voltage becomes uncontrollable because each current is opposite direction. Then the anode voltage becomes discrepant from the equation at deeper modulation and higher duty operation. The relatively large anode current also modify the equation. The $\mathrm{R}_{2}$ should be replaced by $R_{2}{ }^{\prime}=R_{2} / R_{c f f}$, where $R_{c f f}$ is an effective anode resistance. The anode voltage shows also a sag within the pulse-duration, because a charge-up of the capacitor of $0.68 \mu \mathrm{F}$ shown in Figure 1 is starting at the switch-on of the tube. In Figure 2 the typical pulse shapes are shown at an operating condition of 2-MW output. The relatively small sag of the cathode voltage $(2-3 \%)$ is seen, because only one klystron was connected to the power supply. The anode voltage measured to the ground level shows an almost flat shape partially due to the anti-sag of the anode voltage. The rise- and fall-times of anode voltage are explained by the stray capacitor $C_{S 1}$ shown in Figure 1 .

\section{Low Level Control}

A drive power controls the amplitude and the phase of the klystron output in the linear region. A circuit shown in Figure 3 is prepared to process the RF signal. Among the several signals shown in the figure, the "Compensation" signal can be used as a fecdforward signal to compensate the beam loading.
We take into account the filling times of the RFQ $(5.2 \mu \mathrm{sec})$ and the DTL $(14.7 \mu \mathrm{sec})$ in the design of the circuit parameters. The preliminary test of the ALC has been done to stabilize the klystron output at $\sim 1 \mathrm{MW}$ level. Due to the sag of

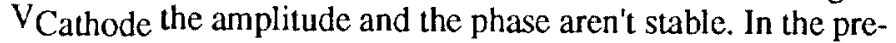
sent parameters, the loop gain can't be large enough because of the oscillation at the leading part of the pulse. The obtaining stability defined as a power change per $1 \mathrm{kV} V_{\text {Cathode }}$ change is $19.5 \mathrm{~kW} / \mathrm{kV}$ at loop-on, while $73.7 \mathrm{~kW} / \mathrm{kV}$ at loop-off. For the PLL, basically same module will be used but the control signal of the phase shift should be bipolar to control \pm phase shift. The final optimization of the parameters should be done with including the cavities.

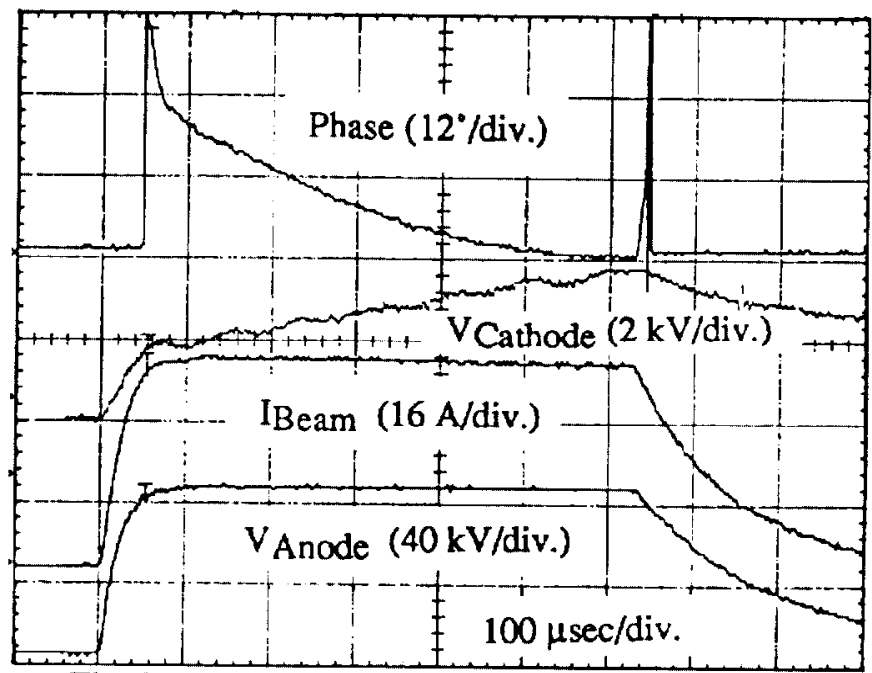

The data are taken at $600 \mu \mathrm{sec}$ pulse-duration, $10 \mathrm{~Hz}$ repetition-rate and $93 \mathrm{kV}$ cathode voltage.

The clamp voltage is $0 \mathrm{kV}$.

Figure 2. Typical Pulse Shapes.

\section{HIGH POWER TEST}

\section{A. Experimental Set up}

Only one klystron was ready to operate at the test. The power generated at the klystron was directed to a dummy load by WR- 1800 waveguide. The dummy load is a coaxial type with a resistive thin film coated on a ceramic pipe as an inner conductor. The input port is the WX-203D flange and the resistor is cooled by a water-flow at the inside-and outside of the

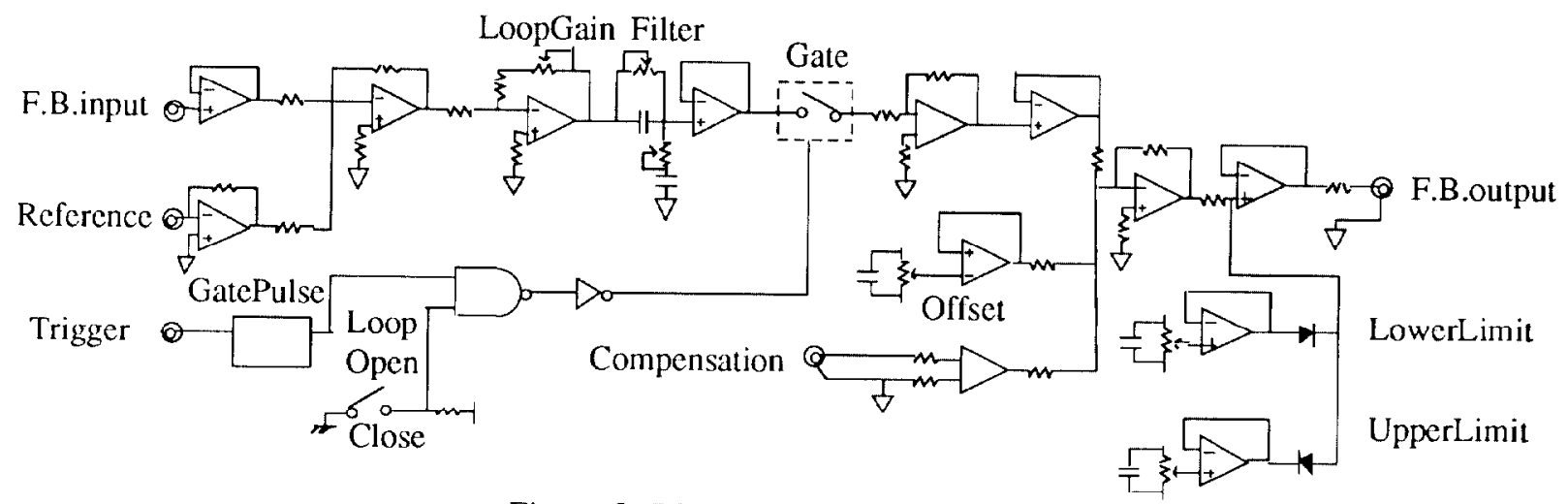

Figure 3. Block Diagram of Feedback Circuit. 
pipe. A space between the water-proof pipe and outer conductor is filled with SF6 gas to prevent the arcing. The load is designed to absorb the RF power of 2-MW output with $65 \mathrm{~kW}$ average power $(650 \mu \mathrm{sec} \times 50 \mathrm{~Hz}$ duty). Two loads were used in parallel at the test, because one load had been damaged by the arcing at $1.6 \mathrm{MW}$ output and $600 \mu \mathrm{sec} \times 50 \mathrm{~Hz}$ duty operation with no SF6 gas filling. The power measured with a directional coupler was checked by a calorimetric measurement at the dummy load with a $\pm 5 \%$ accuracy. The measurements were done at the duty of $600 \mu \mathrm{sec}$ pulse-duration $\times 10 \mathrm{~Hz}$ repetition-rate. The klystron showed relatively stable operation during the test, even though $2 \sim 3$ times crowbar trips had been encountered at an acceptance test.

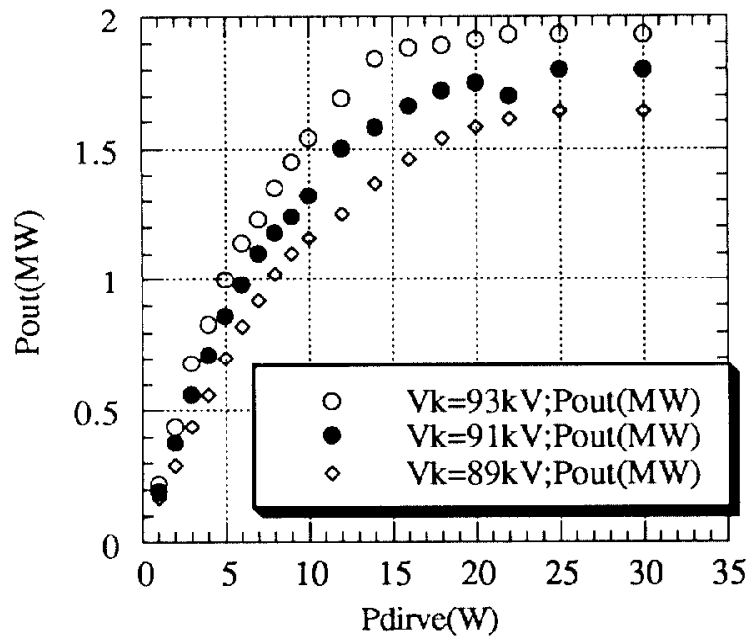

Figure 4. Output Power vs. Drive Power.

\section{B. Performances of the Klystron and Discussion}

In Figure 4 , the output power is shown as a function of the drive-power at three cathode voltages. The observed efficiency at saturation point (drive power $=30 \mathrm{~W}$ ) is $52 \%$ for 2 -MW output at $-93 \mathrm{kV}$ cathode voltage. An observed gun-perveance of the klystron is $1.8 \times 10^{-6} \mathrm{~A} / \mathrm{V}^{3 / 2}$. The phase shift

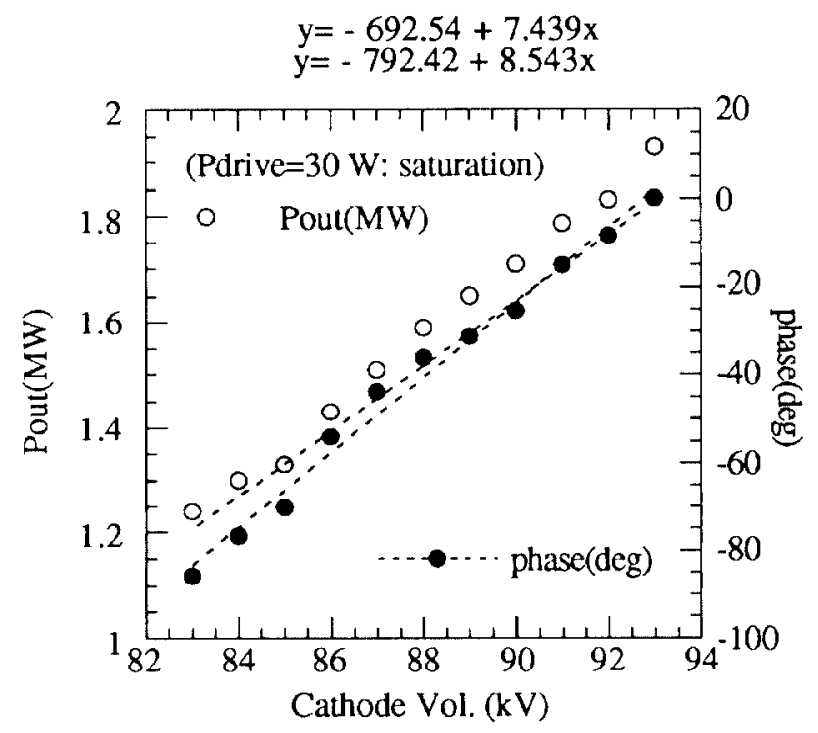

Figure 5. Output Power and Phase Shift.

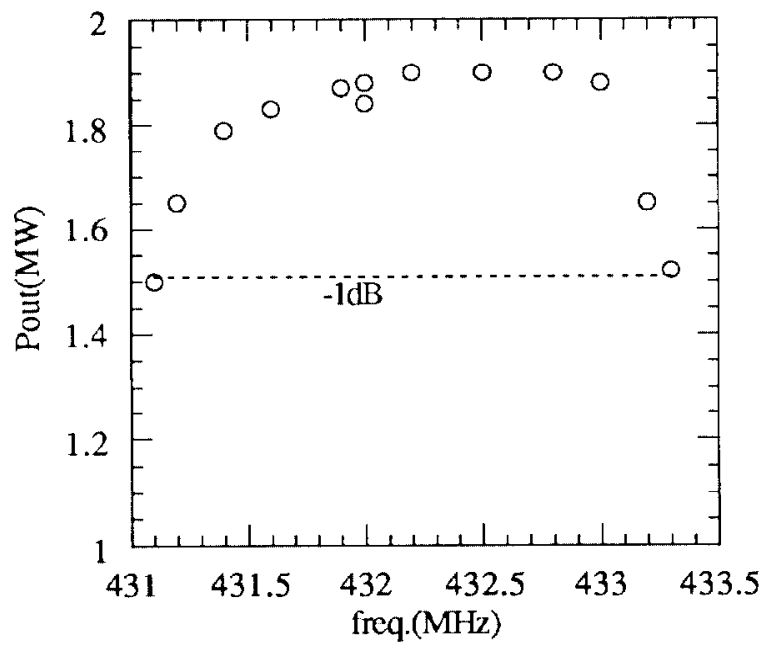

Measured at saturation point (Drive power $=30 \mathrm{~W}$ ).

Figure 6. Band Width at $-1 \mathrm{~dB}$.

measured at saturation are shown in Figure 5 as a function of the cathode voltage. The phase shift per $1 \mathrm{kV}$ cathode voltage change is $\sim 8^{\circ} / \mathrm{kV}$ as shown in the fitting of Figure 5. Because the klystron could be operated without drive power even at the rated cathode- and anode-voltages for generating 2-MW output, the clamp voltage was set to zero at the test; the test was carried out at a deepest modulation. This fact shows the possibility to omit the clamp power supply from the system. At the phase shift measurement, the anode voltage is also changed as a function of cathode voltage as described in II- $D$. Therefor, the measured phase shifts include the effect of the anode voltage changes. The phase shift during the pulse duration exhibited in Figure 2 are consisted with the sag of cathode voltage and the rate of phase shift change; $\sim 18^{\circ}$ corresponding to $\sim 2 \mathrm{kV}$ droop. A band-width for $-1 \mathrm{~dB}$ decrease of output power is shown in Figure 6. More than 2-MHz range is wide enough band width for the RF processing.

\section{SUMMARY}

The first operation of the UHF RF source for the JHP test-linac has been so far accomplished. It may be proved that the constructed system is feasible to supply the well-controlled RF power to the linac. There are still many jobs which should be completed until the start of the linac beam test, such as the stable two klystrons' operation of the system at the nominal output power with nominal duty, the stable RF processing without sacrificing the high loop-gain, etc. The components of the high power transmission system, such as a $\mathrm{Y}$-junction circulator and the dummy load should be tested at high-power and high-duty. The fabrication of the $2 \mathrm{MW}$ circulator has been already finished. The same test of the cavity itself is a most crucial one.

\section{REFERENCES}

[1] Y. Yamazaki and M. Kihara, Proc.1990 Linear Accel. Conf., 543 (1990), Albuquerque, New Mexico. 